\title{
A note on cyclic polynomials in polydiscs
}

\author{
Linus Bergqvist ${ }^{1}$
}

Received: 8 November 2017 / Revised: 10 January 2018 / Accepted: 15 January 2018 /

Published online: 24 January 2018

(C) The Author(s) 2018. This article is an open access publication

\begin{abstract}
We use methods from potential theory and harmonic analysis to show noncyclicity of polynomials on a polydisc whose zero set meets the distinguished boundary along a hypersurface. We also generalize methods used for proving cyclicity for polynomials in two variables with small zero sets to arbitrary dimension. In doing so, we show that in higher dimension, the cyclicity properties of a function do not only depend on the codimension, but also on the orientation of the zero set. Furthermore, we illustrate our results by studying a special class of polynomials. Finally, we use methods from potential theory to prove that our estimates for non-cyclicity are in fact sharp.
\end{abstract}

Keywords Cyclic vectors $\cdot$ Dirichlet-type spaces $\cdot$ Polydiscs

\section{Introduction}

In this article we study cyclic vectors for the shift operators on a range of Hilbert spaces on polydiscs, which among others include the Hardy space and the Dirichlet space. The problem of characterizing cyclic vectors is a difficult one, and already in one variable, the cyclic vectors for the Dirichlet space are not entirely known. In order to better understand the phenomena which determine the cyclicity properties of different functions, we mainly restrict ourselves to studying polynomials in this article. More concretely, we investigate cyclicity properties of polynomials in the so called Dirichlet type spaces on the polydisc. For $\alpha \in \mathbb{R}_{+}^{n}$, we define the Dirichlet type space with parameter $\alpha, D_{\alpha}^{n}$, as the Hilbert space of holomorphic functions on the $n$-dimensional polydisc whose power series expansion

\footnotetext{
$\bowtie$ Linus Bergqvist

linus@math.su.se

1 Department of Mathematics, Stockholm University, Stockholm, Sweden
} 


$$
f\left(z_{1}, \ldots, z_{n}\right)=\sum_{k_{1}=0}^{\infty} \cdots \sum_{k_{n}=0}^{\infty} a_{k_{1}, \ldots, k_{n}} z_{1}^{k_{1}} \ldots z_{n}^{k_{n}}
$$

satisfies

$$
\|f\|_{\alpha}^{2}=\sum_{k_{1}=0}^{\infty} \ldots \sum_{k_{n}=0}^{\infty}\left(k_{1}+1\right)^{\alpha_{1}} \ldots\left(k_{n}+1\right)^{\alpha_{n}}\left|a_{k_{1}, \ldots, k_{n}}\right|^{2}<\infty
$$

When they dimension is obvious from the context, we will simply use the notation $D_{\alpha}$. Furthermore, we denote the multiplier algebra of $D_{\alpha}$ by $M\left(D_{\alpha}\right)$.

Several results regarding the one variables analogue of these spaces can be found in the book [4]. In [5], it was shown that for $\alpha$ with all $\alpha_{i} \leq 2, D_{\alpha}$ is characterized by finiteness of the seminorm given by

$$
\int_{\mathbb{D}^{n}}\left|\partial_{1} \ldots \partial_{n} f\left(z_{1}, \ldots, z_{n}\right)\right|^{2}\left(1-\left|z_{1}\right|^{2}\right)^{1-\alpha_{1}} \ldots\left(1-\left|z_{n}\right|^{2}\right)^{1-\alpha_{n}} d A\left(z_{1}\right) \ldots d A\left(z_{n}\right)
$$

where $A(z)$ is the normalized Lebesgue measure with respect to $\mathbb{D}$. One important property of $D_{\alpha}$ is the fact that they are all reproducing kernel Hilbert spaces, that is, point evaluation is a bounded linear functional.

The shift operators on $D_{\alpha}$ are defined by $S_{i}(f(z))=z_{i} f(z)$, and from their definition and the definition of the norm, we see that they are commuting, bounded linear operators from $D_{\alpha}$ to itself. We say that a function $f$ is cyclic if the smallest invariant subspace of the shift operators which contains $f$ is all of $D_{\alpha}$. This is equivalent to $[f]=D_{\alpha}$, where $[f]=\overline{\operatorname{span}}\left\{z^{k} f(z): k \in \mathbb{N}^{n}\right\}$, where we have used multi-index notation in the previous definition. Since polynomials are dense subsets of all $D_{\alpha}$, we see that a function $f$ is cyclic if and only if there exists a sequence of polynomials $\left\{p_{n}\right\}$ such that $\lim _{n \rightarrow \infty}\left\|p_{n} f-1\right\|_{\alpha}=0$. See for example [3] for details.

In one and two variables, the cyclic polynomials have been completely characterized. In [3] it was shown that all polynomials in one variable with no zeros in $\mathbb{D}$ are cyclic for all $\alpha \leq 1$. In [2] the authors studied isotropic spaces, i.e. spaces with $\alpha_{1}=\cdots=\alpha_{n}$, and they showed that, apart from certain degenerate cases where the polynomial in $\mathbb{C}\left[z_{1}, z_{2}\right]$ was in fact a polynomial in which only one variable was represented, the cyclicity properties were determined by the cardinality of the zero set on $\mathbb{T}^{2}$. They showed that polynomials with only finitely many zeros on $\mathbb{T}^{2}$ are cyclic for all $\alpha \leq 1$, and polynomials with an infinite zero set are cyclic if and only if $\alpha \leq 1 / 2$. In [6], this result was extended to the anisotropic setting, where the authors showed that a polynomial with infinite zero set is cyclic if and only if $|\alpha|=\alpha_{1}+\alpha_{2} \leq 1$. In fact, this was shown to be true even when one $\alpha_{j}$ was negative.

In this article, we generalize some of the methods used in [2] in order to tackle the analogous problem in higher dimension. In particular, a result from [2] which relates non-vanishing Gaussian curvature of the zero set of a function $f$ on $\mathbb{T}^{n}, Z(f) \cap \mathbb{T}^{n}$, to non-cyclicity of $f$ is shown to still be true in arbitrary dimension if $Z(f) \cap \mathbb{T}^{n}$ contains a hypersurface. To avoid repetition, we will assume that $f \neq 0$ in $\mathbb{D}^{n}$, and furthermore, for simplicity we will usually talk about "the zero set of $f$ " instead of " $Z(f) \cap \mathbb{T}^{n}$ " . 
However, whereas in two variables we can only have finite zero sets or zero sets which contain a hypersurface, the situation is more complicated in higher dimensions. It turns out that this method does not yield a complete answer for polynomials whose zero set is not a hypersurface. In order to understand this problem, we instead study a special class of polynomials. The results for this class shows us that two polynomials that both have infinite zero sets will have different cyclicity properties, depending on the dimension of the zero set. Furthermore, in two variables, the only deviations from the general behaviour were the "degenerate polynomials" in which not all variables were represented, but in this article, we show that there are true three-variable polynomials whose cyclicity properties deviate from those of other polynomials whose zero set has the same dimension, essentially as a consequence of the location of the zero set.

Some of the results in this note are contained in the author's 2017 master's thesis at Stockholm University.

\section{Preliminaries}

\subsection{Some lemmas regarding cyclicity}

The following lemmas will be very valuable for understanding cyclicity properties of functions, and especially polynomials.

Lemma 1 Let $f$ and $g \in D_{\alpha}$ be polynomials such that $|f(z)|>|g(z)|$ for $z \in \mathbb{D}^{n}$. If $g$ is cyclic in $D_{\alpha}$ for some $\alpha \in \mathbb{R}_{+}^{n}$, then $f$ is cyclic in $D_{\alpha}$.

The above theorem is merely a special case of the Corollary 1 on page 281 in [3] which concerns cyclicity in Dirichlet spaces. The proof for weighted Dirichlet spaces in several variables is analogous.

Lemma 2 Let $f \in D_{\alpha}$ and $g \in M\left(D_{\alpha}\right)$, then $g f \in D_{\alpha}$ is cyclic if and only if both $f$ and $g$ are cyclic.

The proof is given in Proposition 8 of [3].

The above lemma is very useful when working with polynomials. For example, it shows us that if $p\left(z_{1}, \ldots z_{n}\right)^{n}$ is cyclic, then so is the polynomial $p\left(z_{1}, \ldots, z_{n}\right)$.

The proof for the following lemma is completely analogous to the two dimensional counterpart, the proof of which is given in Proposition 2.4 of [1].

Lemma 3 Let $f\left(z_{1}, \ldots, z_{n}\right)=g\left(z_{1}, \ldots, z_{k}\right) h\left(z_{k+1}, \ldots, z_{n}\right)$ for $g \in D_{\alpha^{\prime}}^{k}$ and $h \in$ $D_{\alpha^{\prime \prime}}^{n-k}$. Then $f$ is cyclic in $D_{\left(\alpha^{\prime}, \alpha^{\prime \prime}\right)}$ if and only if $g$ is cyclic in $D_{\alpha^{\prime}}^{k}$ and $h$ is cyclic in $D_{\alpha^{\prime \prime}}^{n-k}$.

The previous two lemmas are especially important when working with polynomials. For example, they imply that it is sufficient to understand the cyclicity properties of irreducible polynomials. Also, when working with polynomials in several variables, for example three variables, it sufficies to understand the cyclicity properties of polynomials which are really polynomials in all three variables, since the cyclicity properties of one variable and two variable polynomials are known. 


\subsection{Möbius transformations and the type of a set}

For simplicity, we will later study polynomials in three variables. At that point we will need certain results which are related to Möbius transformations in three variables.

The automorphism group of the tridisc consists of rotations, permutations of variables, and Möbius transformations

$$
m_{a, b, c}:(x, y, z)=\left(\frac{a-x}{1-\bar{a} x}, \frac{b-y}{1-\bar{b} y}, \frac{c-z}{1-\bar{c} z}\right),
$$

with $a, b, c \in \mathbb{D}$. By using the integral norm of $D_{\alpha}$, it can be shown that composition with any Möbius transformation defines a bounded operator from $D_{\alpha}$ to itself. In this article, this is mainly important since it implies that if a function $f$ is cyclic then every function of the form $f \circ m_{a, b, c}$ is also cyclic. To see this, simply note that

$$
\left\|\left(p_{n} \circ m_{a, b, c}\right)\left(f \circ m_{a, b, c}\right)-1\right\|_{\alpha} \leq\left\|m_{a, b, c}\right\|\left\|p_{n} f-1\right\|_{\alpha},
$$

where $\left\|m_{a, b, c}\right\|$ denotes the operator norm.

Furthermore, this implies that $f$ is non-cyclic if any $f \circ m_{a, b, c}$ is non-cyclic.

Composition with any rotation or permutation is in fact an isometry, and so a function is cyclic if and only if any such composite is cyclic. This is a major convenience as it allows us to, without loss of generality, study local behaviour at any suitable point.

We will use this definition from section VIII3.2 in [9].

Definition 1 Let $S \subset \mathbb{T}^{n}$ be a smooth $m$-manifold. Let $\phi: I^{m} \rightarrow \mathbb{T}^{n}$ be a smooth parametrization, where $I \subset \mathbb{R}$ is an interval. We define the type of a point $\xi=\phi(x)$ as the smallest $\tau$ such that for all unit vectors $\eta \in \mathbb{R}^{n}$ there exists a multi-index $k \in \mathbb{N}^{m}$ with $|k| \leq \tau$ such that

$$
\left[\frac{d^{k} \phi}{d t^{k}} \cdot \eta\right]_{t=x} \neq 0
$$

We say that $S$ has type $\tau$ if the maximum of the types of $\xi \in S$ is $\tau$.

In particular, having type 2 is the same as having non-vanishing Gaussian curvature.

For a function $f \in D_{\alpha}$, it will later be proved that there is a strong connection between $f$ being cyclic and $Z(f) \cap \mathbb{T}^{3}$ having type 2 . Therefore, it is especially important to understand when a set which can locally be parametrized by $\phi(x)=$ $(x, g(x), h(x))$ or $\phi(x, y)=(x, y, g(x, y))$ has type 2 . It will also be very useful to know that even if a curve or a surface does not have type 2, we can still, in certain circumstances, apply a Möbius transformation in order to obtain a new curve or surface which does have type 2 . This new curve will be the zero set of another function whose cyclicity properties can be related to those of $f$.

Now, given a function $f$ such that $Z(f) \cap \mathbb{T}^{3}$ is locally parametrized by $\phi(x, y)=$ $(x, y, g(x, y))$, we know that the zero set does not have type 2 at the point $(0,0, g(0,0))$ if and only if there is some $\eta \in S^{3}$ such that

$$
\eta_{1}+g_{x}^{\prime}(0,0) \eta_{3}=\eta_{2}+g_{y}^{\prime}(0,0) \eta_{3}=0
$$


and

$$
g_{x x}^{\prime \prime}(0,0) \eta_{3}=g_{y y}^{\prime \prime}(0,0) \eta_{3}=g_{x y}^{\prime \prime}(0,0) \eta_{3}=0 .
$$

This is possible if and only if all second derivatives of $g(x, y)$ vanish.

However, even if all second order partial derivatives vanish, we can still precompose $f$ with a Möbius transformation $m_{a, b, 0}$ in order to obtain a new function $f \circ m_{a, b, 0}$. A piece of the zero set of this new function will be parametrized by

$$
\left(\arg m_{a}\left(e^{i x}\right), \arg m_{b}\left(e^{i y}\right), g(x, y)\right) .
$$

For convenience we denote $\arg m_{a}\left(e^{i x}\right)$ by $\psi_{a}(x)$. One can compute that $\psi_{a}^{\prime}(0)>0$ and $\psi_{a}^{\prime \prime}(0) \neq 0$ as long as $\operatorname{Im}(a) \neq 0$. For suitable choices of $a$ and $b$ we see that this new surface fails to have type 2 if and only if there is some $\theta \in S^{3}$ such that

$$
\psi_{a}^{\prime}(0) \theta_{1}+g_{x}^{\prime}(0,0) \theta_{3}=\psi_{b}^{\prime}(0) \theta_{2}+g_{y}^{\prime}(0,0) \theta_{3}=0
$$

and

$$
\psi_{a}^{\prime \prime}(0) \theta_{1}+g_{x x}^{\prime \prime}(0,0) \theta_{3}=\psi_{b}^{\prime \prime}(0) \theta_{2}+g_{y y}^{\prime \prime}(0,0) \theta_{3}=g_{x y}^{\prime \prime}(0,0) \theta_{3}=0 .
$$

But as noted before, if our original surface did not have type 2, then all second derivatives of $g$ must vanish. Thus, in order for the second family of equations to hold both $\theta_{1}$ and $\theta_{2}$ must vanish. Together with the first family of equations this implies that the new surface does not have type 2 if and only if all first order and second order derivatives of $g$ vanish at the origin.

If however a piece of $Z(f) \cap \mathbb{T}^{3}$ can be parametrized locally by $(x, g(x), h(x))$, then it has type 2 if and only if there is some $\eta \in S^{3}$ for which

$$
\eta_{1}+g^{\prime}(x) \eta_{2}+h^{\prime}(x) \eta_{3}=0
$$

and

$$
g^{\prime \prime}(x) \eta_{2}+h^{\prime \prime}(x) \eta_{3}=0 .
$$

If this surface does not have type 2, then we can once again apply a Möbius transformation, $m_{a, 0,0}$ in order to obtain a new function and a new zero set. This new zero set has type 2 if and only if there exist $\theta \in S^{3}$ such that

$$
\psi_{a}^{\prime}(0) \theta_{1}+g^{\prime}(x) \theta_{2}+h^{\prime}(x) \theta_{3}=0
$$

and

$$
\psi_{a}^{\prime \prime}(0) \theta_{1}+g^{\prime \prime}(x) \theta_{2}+h^{\prime \prime}(x) \theta_{3}=0 .
$$

Unfortunately, there are several examples of when such $\theta \in S^{3}$ exist: for example, if both $g^{\prime \prime}(0)$ and $h^{\prime \prime}(0)=0$. 
At this point, we wish to point out some similarities and differences between the above discussion and the two variable counterpart in Section 2.3 in [2]. In two variables, the case of a curve coincides with that of a hypersurface. The results in three variables largely coincide with those of hypersurfaces in two variables, namely it is almost always possible to apply Möbius transformations in order to obtain a set of type 2 . However, in three variables we also have a possibility which does not exist in two variables, namely when the zero set is locally a curve. The above calculations show that we can not in general expect to be able to apply the methods for determining non-cyclity that were used in [2], so we must instead find other methods.

\subsection{Riesz capacity}

We will later use known results which relate existence of measures of finite Riesz energy that are supported on $Z(f) \cap \mathbb{T}^{n}$ to non-cyclicity of $f$. In this article we define the Riesz potential with parameter $\alpha \in(0,1)^{n}, h_{\alpha}$, as

$$
h_{\alpha}\left(x_{1}, \ldots, x_{n}\right)=1 /\left|1-e^{i x_{1}}\right|^{1-\alpha_{1}} \ldots 1 /\left|1-e^{i x_{n}}\right|^{1-\alpha_{n}} .
$$

One important lemma regarding the Riesz energy of measures is the following.

Lemma 4 Let $h_{\alpha}$ be the Riesz potential with parameter $\alpha \in(0,1)^{n}$, we have that

$$
\begin{aligned}
I_{h_{\alpha}}[\mu] & =\int_{\mathbb{T}^{n}} \int_{\mathbb{T}^{n}} \frac{1}{\left|e^{i x_{1}}-e^{i y_{1}}\right|^{1-\alpha_{1}} \ldots\left|e^{i x_{n}}-e^{i y_{n}}\right|^{1-\alpha_{n}}} d \mu(x) d \mu(y) \\
& =\sum_{k_{1}=-\infty}^{\infty} \cdots \sum_{k_{n}=-\infty}^{\infty} \widehat{h_{\alpha}}(k)|\hat{\mu}(k)|^{2} .
\end{aligned}
$$

It is known that the Fourier coefficients of $h_{\alpha}$ satisfy

$$
c\left(\left|k_{1}\right|+1\right)^{-\alpha_{1}} \ldots\left(\left|k_{n}\right|+1\right)^{-\alpha_{n}} \leq \widehat{h_{\alpha}}(k) \leq C\left(\left|k_{1}\right|+1\right)^{-\alpha_{1}} \ldots\left(\left|k_{n}\right|+1\right)^{-\alpha_{n}} .
$$

The summation formula for the Riesz $\alpha$-energy will later be used in order to show that certain sets have non-zero Riesz capacity.

\section{Cyclicity in $n$ variables}

\subsection{Small zero sets}

In $[2,6]$, Lemma 1 was used to show cyclicity for polynomials in $\mathbb{C}[x, y]$ with finite zero set. The idea was to use Łojasiewicz's inequality, Lemma 1, combined with knowledge of the cyclicity properties of one-variable polynomials. In two variables not much more could be said using this idea. But in higher dimensions we observe that polynomials of the form $c_{j}-x_{j}$ have very large zero sets. By combining this with the above idea we can show the following. 
Theorem 1 Let $f$ be a polynomial with no zeros in $\mathbb{D}^{n}$. If $Z(f) \cap \mathbb{T}^{n}$ is contained in a finite union of coordinate hyperplanes of $\mathbb{T}^{n}$, then $f$ is cyclic for all $\alpha \in(0,1]^{n}$.

Proof Denote by $S$ the union of coordinate planes, $\bigcup_{j}\left\{z: z_{j}=c_{j}\right\}$, with $c_{j} \in \mathbb{T}$ which contain $Z(f) \cap \mathbb{T}^{n}$. By Łjasiewicz's inequality (as quoted in [7]) we know that there exists $C>0$ and $q \in \mathbb{N}$ such that

$$
\begin{aligned}
& |f(z)|>c \cdot \operatorname{dist}(z, Z(f))^{q}>C \cdot \operatorname{dist}(z, S)^{q} \\
& \quad=C \cdot \min \left\{\left|z_{j}-c_{j}\right|\right\}^{q} \geq C \cdot\left(\prod_{i} \frac{\left|z_{j}-c_{j}\right|}{2}\right)^{q} .
\end{aligned}
$$

By applying Lemmas 1 and 2 this shows us that $f$ is cyclic whenever the polynomials $z_{j}-c_{j}$ are cyclic, which is for all $\alpha \leq 1$.

For $n=2$, only one-variable polynomials and polynomials with finite zero set satisfy the assumptions of the above Theorem. But as we will see, this is no longer true in higher dimensions.

\subsection{Large zero sets}

The cornerstone in our proofs regarding non-cyclicity is the following theorem, which relates properties of $Z(f) \cap \mathbb{T}^{n}$ to non-cyclicity of $f$.

Theorem 2 If $f \in D_{\alpha}$ for $\alpha \in(0,1)^{n}$, and $Z(f) \subset \mathbb{T}^{n}$ has positive Riesz capacity, then $f$ is not cyclic.

The proof is largely analogous to Brown and Shields' proof for the Dirichlet space in the disc, with only minor differences related to using Riesz capacity instead of logarithmic capacity. See Theorem 5 in [3] for the complete proof. For a good outline of the proof idea in two variables we refer the reader to Proposition 4.2 in [1]. Later on we will use a partial result from the proof of the above theorem, so for convenience we state that partial result as a lemma.

Lemma 5 If $f \in D_{\alpha}$ has the property that $Z(f) \cap \mathbb{T}^{n}$ supports a measure $\mu$ whose Cauchy transform $C[\mu]$ lies in $D_{-\alpha}$, then $f$ is not cyclic in $D_{\alpha}$.

Our next goal is to relate certain geometric properties of $Z(f) \cap \mathbb{T}^{n}$ to existence of measures with finite Riesz energy. In order to do so we need the following theorem, the proof of which can be found on page 348, Theorem 1 and page 351 Theorem 2 in [9].

Theorem 3 Let $S \subset \mathbb{T}^{n}$ be a locally smooth m-manifold of finite type $\tau \in \mathbb{N}$ and let $\sigma$ be the measure on $S$ induced by pulling back to the Lebesgue measure using the parametrization of $S$. If $\mu$ is a measure of the form $d \mu(x)=\phi(x) d \sigma(x), x \in S \subset \mathbb{T}^{n}$, where $\phi(x)$ is a non-negative smooth function with compact support (defined on $S$ ), then there exists a constant $C>0$ such that

$$
\left|\hat{\mu}\left(k_{1}, \ldots, k_{n}\right)\right| \leq C\left(k_{1}^{2}+\cdots+k_{n}^{2}\right)^{-1 / 2 \tau}, \quad k_{1}, \ldots, k_{n} \in \mathbb{Z} \backslash\{0\} .
$$


If we have a smooth hypersurface of non-vanishing Gaussian curvature, that is it has type 2, then

$$
\left|\hat{\mu}\left(k_{1}, \ldots, k_{n}\right)\right|^{2} \leq C\left(k_{1}^{2}+\cdots+k_{n}^{2}\right)^{(1-n)}, \quad k_{1}, \ldots, k_{n} \in \mathbb{Z} \backslash\{0\} .
$$

Theorem 4 Assume that $f \in D_{\alpha}$ for $\alpha \in(0,1)^{n}$, is such that $Z(f) \cap \mathbb{T}^{n}$ contains a locally smooth $m$-manifold of finite type $\tau$. Then $f$ is not cyclic in $D_{\alpha}$ for any $\alpha$ for which $|\alpha|>n-2 / \tau$. If it contains a locally smooth hypersurface of type 2 , then $f$ is not cyclic in $D_{\alpha}$ for any $\alpha$ for which $|\alpha|>1$.

We only give the proof of the statement for a general $m$-manifold, the proof of the statement for the hypersurface is completely analogous if one uses the second inequality in Theorem 3 instead of the first inequality.

Proof Since $S \subset Z(f)$ we know that the Riesz capacity of $S$ is less than or equal to the Riesz capacity of $Z(f)$. So if we can show that $S$ supports a measure of finite $\alpha$-energy for $|\alpha|>n-2 / \tau$, and thus show that $S$ has positive Riesz capacity, then it follows that $Z(f)$ has positive Riesz capacity. By applying Theorem 2 the statement follows.

That $S$ supports such a measure will be shown by using Theorem 3 together with Lemma 4, since these statements prove that all absolutely continuous measures have Fourier coefficients which decay quickly.

Let $\mu$ be any absolutely continuous measure on $F$. From Lemma 4 with $K=h_{\alpha}$ and the bound on the Fourier coefficients of the Riesz capacity from Eq. 2, we have

$$
I_{h_{\alpha}}[\mu] \leq C \sum_{k_{n}=-\infty}^{\infty} \cdots \sum_{k_{1}=-\infty}^{\infty} \frac{|\hat{\mu}(k)|^{2}}{\left(\left|k_{n}\right|+1\right)^{\alpha_{n}} \ldots\left(\left|k_{1}\right|+1\right)^{\alpha_{1}}}
$$

$\mu$ is a probability measure, $|\hat{\mu}(0, \ldots, 0)|^{2}=1$, and so, from Theorem 3 and the assumptions on $\mu$, we have that

$$
\text { (3) } \leq C\left(1+\sum_{k_{n}=-\infty}^{\infty} \cdots \sum_{k_{1}=-\infty}^{\infty} \frac{1}{\left(\left|k_{n}\right|+1\right)^{\alpha_{n}} \ldots\left(\left|k_{1}\right|+1\right)^{\alpha_{1}}\left(k_{1}^{2}+\cdots+k_{n}^{2}\right)^{1 / \tau}}\right) \text {, }
$$

where $k_{1}, \ldots, k_{n}$ are not all equal to zero in the above series. The above expression is finite if and only if the series is finite. By using that the summand is even in each $k_{i}$, we have that

$$
\begin{aligned}
& \sum_{k_{n}=-\infty}^{\infty} \cdots \sum_{k_{1}=-\infty}^{\infty} \frac{1}{\left(\left|k_{n}\right|+1\right)^{\alpha_{n}} \ldots\left(\left|k_{1}\right|+1\right)^{\alpha_{1}}\left(k_{1}^{2}+\cdots+k_{n}^{2}\right)^{1 / \tau}} \\
& \leq 2^{n} \sum_{k_{n}=0}^{\infty} \cdots \sum_{k_{1}=0}^{\infty} \frac{1}{\left(\left|k_{n}\right|+1\right)^{\alpha_{n}} \ldots\left(\left|k_{1}\right|+1\right)^{\alpha_{1}}\left(k_{1}^{2}+\cdots+k_{n}^{2}\right)^{1 / \tau}} .
\end{aligned}
$$


Note that we do not necessarily have equality since terms with at least one $k_{i}$ equal to 0 are counted several times in the second expression. We can now bound the above series by

$2^{n} \sum_{\sigma \in \operatorname{Sym}(n)} \sum_{k_{\sigma(n)}=1}^{\infty} \sum_{k_{\sigma(n-1)}=0}^{k_{\sigma(n)}} \cdots \sum_{k_{\sigma(1)}=0}^{k_{\sigma(2)}} \frac{1}{\left(\left|k_{n}\right|+1\right)^{\alpha_{n}} \ldots\left(\left|k_{1}\right|+1\right)^{\alpha_{1}}\left(k_{1}^{2}+\cdots+k_{n}^{2}\right)^{1 / \tau}}$,

where $\operatorname{Sym}(n)$ is the symmetric group of $n$ symbols, i.e. the group of permutations. Note that we again do not have equality, since terms with $k_{i}=k_{j}$ for $i \neq j$ will be counted several times in the last series, e.g. all terms with $k_{1}=k_{2}$ will be covered by both $I d \in \operatorname{Sym}(n)$ and (12) $\in \operatorname{Sym}(n)$.

Note that $k_{\sigma(n)}$ cannot be zero as this would force all $k_{i}$ 's to be zero.

Since the symmetric group is finite, we are done if we can show that

$$
\sum_{k_{\sigma(n)}=1}^{\infty} \sum_{k_{\sigma(n-1)}=0}^{k_{\sigma(n)}} \cdots \sum_{k_{\sigma(1)}=0}^{k_{\sigma(2)}} \frac{1}{\left(\left|k_{n}\right|+1\right)^{\alpha_{n}} \ldots\left(\left|k_{1}\right|+1\right)^{\alpha_{1}}\left(k_{1}^{2}+\cdots+k_{n}^{2}\right)^{1 / \tau}}<\infty
$$

is finite for $\alpha>1-2 / n \tau$ for each $\sigma$. The calculations are completely analogous for each $\sigma \in \operatorname{Sym}(n)$, and so we will only show this for $\sigma=I d$, i.e.

$$
\sum_{k_{n}=1}^{\infty} \sum_{k_{n-1}=0}^{k_{n}} \cdots \sum_{k_{1}=0}^{k_{2}} \frac{1}{\left(\left|k_{n}\right|+1\right)^{\alpha_{n}} \cdots\left(\left|k_{1}\right|+1\right)^{\alpha_{1}}\left(k_{1}^{2}+\cdots+k_{n}^{2}\right)^{1 / \tau}} .
$$

That this is true can be seen in the following way.

$$
\begin{aligned}
\text { (4) } & \leq C \sum_{k_{n}=1}^{\infty} \sum_{k_{n-1}=0}^{k_{n}} \cdots \sum_{k_{1}=0}^{k_{2}} \frac{1}{\left(\left|k_{n}\right|+1\right)^{\alpha_{n}} \ldots\left(\left|k_{1}\right|+1\right)^{\alpha_{1}} k_{n}^{2 / \tau}} \\
& =C \sum_{k_{n}=1}^{\infty} k_{n}^{-2 / \tau} \sum_{k_{n-1}=0}^{k_{n}} \cdots \sum_{k_{2}=0}^{k_{3}} \frac{1}{\left(\left|k_{n}\right|+1\right)^{\alpha_{n}} \ldots\left(\left|k_{2}\right|+1\right)^{\alpha_{2}}} \sum_{k_{1}=0}^{k_{2}} \frac{1}{\left(\left|k_{1}\right|+1\right)^{\alpha_{1}}}
\end{aligned}
$$

By applying the inequality $\sum_{k=0}^{l}(k+1)^{(m-1)-m \alpha} \leq C(l+1)^{m-m \alpha}$, inductively to (6) we get that

$$
\begin{aligned}
\text { (6) } & \leq C \sum_{k_{n}=1}^{\infty} k_{n}^{-2 / \tau} \sum_{k_{n-1}=0}^{k_{n}} \cdots \sum_{k_{2}=0}^{k_{3}} \frac{\left(k_{2}+1\right)^{1-\alpha_{1}}}{\left(\left|k_{n}\right|+1\right)^{\alpha_{n}} \ldots\left(\left|k_{2}\right|+1\right)^{\alpha_{2}}} \\
& =C \sum_{k_{n}=1}^{\infty} k_{n}^{-2 / \tau} \sum_{k_{n-1}=0}^{k_{n}} \cdots \sum_{k_{3}=0}^{k_{4}} \frac{1}{\left(\left|k_{n}\right|+1\right)^{\alpha_{n}} \ldots\left(\left|k_{3}\right|+1\right)^{\alpha_{3}}} \sum_{k_{2}=0}^{k_{3}}\left(k_{2}+1\right)^{1-\left(\alpha_{1}+\alpha_{2}\right)}
\end{aligned}
$$




$$
\begin{aligned}
& \leq C \sum_{k_{n}=1}^{\infty} k_{n}^{-2 / \tau} \sum_{k_{n-1}=0}^{k_{n}} \cdots \sum_{k_{3}=0}^{k_{4}} \frac{\left(k_{3}+1\right)^{2-\left(\alpha_{1}+\alpha_{2}+\alpha_{3}\right)}}{\left(\left|k_{n}\right|+1\right)^{\alpha_{n}} \ldots\left(\left|k_{4}\right|+1\right)^{\alpha_{4}}} \\
& \vdots \\
& \leq C \sum_{k_{n}=1}^{\infty} k_{n}^{-2 / \tau}\left(k_{n}+1\right)^{(n-1)-|\alpha|} \leq C \sum_{k_{n}=1}^{\infty}\left(k_{n}+1\right)^{(n-1)-|\alpha|-2 / \tau} .
\end{aligned}
$$

The last sum is finite if and only if $(n-1)-|\alpha|-2 / \tau<-1 \Longleftrightarrow n-2 / \tau<|\alpha|$, which is what we want to show.

\section{Polynomials in three variables}

For simplicity, we will now turn our attention to polynomials in three variables. Here we will show that the above theorem can in fact be applied "very often" for hypersurfaces.

Theorem 5 Let $f \in \mathbb{C}[x, y, z]$ be a polynomial with no zeros in $\mathbb{D}^{3}$, and in which all variables are represented. If $Z(f) \cap \mathbb{T}^{3}$ contains a hypersurface $S$ which is not contained in some coordinate plane of $\mathbb{T}^{3}$, then $f$ is not cyclic in $D_{\alpha}^{3}$ for any $\alpha \in(0,1)^{3}$ with $|\alpha|>1$.

Proof We have two possibilities. Either $S$ contains a point of type 2, or it does not contain a point of type 2 . If $S$ has type 2 , then the statement follows immediately from Theorem 4.

Without loss of generality, let a part of $S$ be parametrized by $(x, y, g(x, y))$. From the discussion in Sect. 2.2, we can apply Möbius transformations to the parameters in the parametrization in order to obtain a new surface that is of type 2 at the point $p$, as long as some partial derivative of $g$ is non-vanishing at some point in the parametrized part of $S$, i.e. $g$ is not constant. But by assumption, $S$ is not contained in some coordinate plane, and so we know that $g$ has a non-vanishing partial derivative.

After applying a Möbius transformation, we obtain a new surface which is a parametrization of a part of $Z(f) \cap \mathbb{T}^{3}$ for the function $f \circ m_{a, b, 0}$. By applying Theorem 4, we can conclude that $f \circ m_{a, b, 0}$ is not cyclic for any $|\alpha|>1$. This in turn implies that $f$ is not cyclic, since assuming that $f$ is cyclic, then there exists a sequence of polynomials $p_{n}$ for which

$$
\lim _{n \rightarrow \infty}\left\|p_{n} f-1\right\|_{\alpha}=0
$$

But this is not possible since this would imply that

$$
\left\|\left(p_{n} \circ m_{a, b, 0}\right) \cdot\left(f \circ m_{a, b, 0}\right)-1\right\|_{\alpha} \leq\left\|m_{a, b, 0}\right\|\left\|p_{n} f-1\right\|_{\alpha},
$$

where the right hand side tends to zero as $n \rightarrow \infty$. But by approximating $p_{n} \circ m_{a, b, 0}$ by polynomials $q_{n}$, this implies that 


$$
\lim _{n \rightarrow \infty}\left\|q_{n} f \circ m_{a, b, 0}-1\right\|_{\alpha}=0
$$

and so $f \circ m_{a, b, 0}$ would be cyclic, a contradiction.

Note that the case where $Z(f) \cap \mathbb{T}^{3}$ is contained in a coordinate plane was already covered in Theorem 1.

\subsection{A special class of polynomials}

In the previous subsection we showed that methods, similar to those used in [2] for proving non-cyclicity of two-variable polynomials, worked well for proving noncyclicity of three-variable polynomials whose zero set in the 3-torus contains a hypersurface. However, from the discussion in Sect. 2.2, it is clear that the situation is more delicate for polynomials whose zero set meets the 3-torus along a curve. In this subsection, we will instead focus on a special class of polynomial and try to better understand their cyclicity properties.

Consider all polynomials in $\mathbb{C}[x, y, z]$ of the form

$$
p(x, y, z)=1-\sum_{i=1}^{l} d_{i} x^{a_{i}} y^{b_{i}} z^{c_{i}},
$$

where $a_{i}, b_{i}, c_{i} \in \mathbb{N}, \operatorname{Im}\left(d_{i}\right)=0, d_{i}>0$ and $\sum d_{i}=1$. None of these polynomials have any zeros in $\mathbb{D}^{3}$, and so they are good candidates for being cyclic. Since the cyclicity properties are completely understood for one-, and two-variable polynomials, as well as for polynomials with a finite zero set, we will only be interested in polynomials of the above form in which all three variables are represented and whose zero set is infinite.

By simple arguments from linear algebra, one can conclude that every zero set of polynomial of the form (7) will also be the zero set of a polynomial of the form

$$
1-x^{a} y^{b} z^{c} \quad \text { or } 2-x^{a} y^{b}-x^{a} z^{c} \text { or } 2-x^{a} y^{b}-z^{c}
$$

or some composite of such a polynomial with an element in the automorphism group of the tridisc. For simplicity we will henceforth study polynomials of the form (8), which we will refer to as flat polynomials. Since we are only interested in true threevariable polynomials, we assume that $a, b, c \neq 0$. The first class of flat polynomials will have a hyperplane with rational coefficients as their zero set, and the last two will have a line with rational coefficients as their zero set.

One method for showing cyclicity for such polynomials is by comparing them to polynomials in fewer variables whose cyclicity properties are known, namely the polynomials $1-z$ and $2-x-y$, both of which are known to be cyclic for all $\alpha \leq 1$ (finite zero set). Note that by Theorem 1 , we already know that $2-x^{a} y^{b}-z^{c}$ is cyclic for all $\alpha \in(0,1)^{3}$.

Theorem 6 All flat polynomials whose zero set on the 3-torus is parametrized by a (finite) collection of curves which do not lie in a coordinate plane of $\mathbb{T}^{3}$ are cyclic for all $\alpha \in(0,1]^{3}$ for which $\alpha_{1}+\alpha_{2}$ and $\alpha_{1}+\alpha_{3} \leq 1$. 
Proof Let $\alpha \in(0,1]^{3}$ be such that $\alpha_{1}+\alpha_{2}$ and $\alpha_{1}+\alpha_{3} \leq 1$. Denote by $L_{a, b, c}(f)$ the operation which takes $f(s, t)$ to $f\left(x^{a} y^{b}, x^{a} z^{c}\right)$. We will show that $\left\|L_{a, b, c}(f)\right\|_{\alpha} \leq$ $C\|f\|_{\alpha^{\prime}}$, where $\alpha^{\prime}=\left(\alpha_{1}+\alpha_{2}, \alpha_{1}+\alpha_{3}\right)$.

For $f(s, t)=\sum_{k, l \in \mathbb{N}} \hat{f}(k, l) s^{k} t^{l}$, we have that

$$
L_{a, b, c}(f)(x, y, z)=\sum_{p_{1}, p_{2}, p_{3} \in \mathbb{N}} A_{p} x^{p_{1}} y^{p_{2}} z^{p_{3}}=\sum_{k, l \in \mathbb{N}} \hat{f}(k, l) x^{a k+a l} y^{b k} z^{c l},
$$

and so $A_{p}=\hat{f}(k, l)$ if $\left(p_{1}, p_{2}, p_{3}\right)=(a k+a l, b k, c l)$. It follows that

$$
\begin{aligned}
\left\|L_{a, b, c}(f)\right\|_{\alpha}^{2} & =\sum_{p \in \mathbb{N}^{3}}\left|A_{p}\right|^{2}\left(1+p_{1}\right)^{\alpha_{1}}\left(1+p_{2}\right)^{\alpha_{2}}\left(1+p_{3}\right)^{\alpha_{3}} \\
& =\sum_{k, l \in \mathbb{N}}|\hat{f}(k, l)|^{2}(1+a k+a l)^{\alpha_{1}}(1+b k)^{\alpha_{2}}(1+c l)^{\alpha_{3}} \\
& \leq C(a, b, c) \sum_{k, l \in \mathbb{N}}|\hat{f}(k, l)|^{2}(1+k)^{\alpha_{1}+\alpha_{2}}(1+l)^{\alpha_{1}+\alpha_{3}} \\
& =C(a, b, c)\|f\|_{\alpha^{\prime}}^{2} .
\end{aligned}
$$

By noting that $L_{a, b, c}(2-s-t)(x, y, z)=2-x^{a} y^{b}-x^{a} z^{c}$, and using that $2-s-t$ is cyclic for all $\alpha \in(0,1]^{2}$, we know that there is a sequence of polynomials $p_{n}$ such that

$$
\lim _{n \rightarrow \infty}\left\|(2-s-t) p_{n}(s, t)-1\right\|_{(1,1)}=1
$$

By using the above norm inequality and setting $q_{n}=L_{a, b, c}\left(p_{n}\right)$, we see that

$$
\lim _{n \rightarrow \infty}\left\|q_{n} L_{a, b, c}(2-s-t)-1\right\|_{\alpha}=0
$$

and so $2-x^{a} y^{b}-x^{a} z^{c}$ is cyclic for $\alpha$ with the described properties.

By using the same method as above but instead comparing $1-x^{a} y^{b} z^{c}$ to $1-s$, we can conclude that the polynomials in our class whose zero set on $\mathbb{T}^{3}$ is a hypersurface will be cyclic for all $\alpha$ such that $|\alpha| \leq 1$. By combining this with Theorem 5 , we obtain the following.

Theorem 7 Every flat polynomial whose zero set on $\mathbb{T}^{3}$ is a hypersurface not entirely contained in a finite collection of coordinate planes is cyclic in $D_{\alpha}, \alpha \in(0,1)^{3}$, if and only if $|\alpha| \leq 1$.

As previously noted, if the zero set of a polynomial is entirely contained in a coordinate plane, then that polynomial is cyclic for all $\alpha \in(0,1]^{3}$. We also note that there are true three-variable polynomials with this property, for example the polynomial $2-y-x z$. One might expect that this is always the case for polynomials whose zero set is a curve, but as the next example will show, this is not true. In fact, all remaining 
flat polynomials are non-cyclic for all $\alpha \in(0,1]^{3}$ with $|\alpha|>2$. This will be shown by finding a measure supported on $Z(f) \cap \mathbb{T}^{3}$ whose Cauchy transform lies in $D_{\alpha}$ for all $\alpha$ with $|\alpha| \leq-2$, and then applying Lemma 5 .

The measure we have in mind is a simple one, namely the probability measure $\mu$ that has constant density everywhere on the parametrized piece of $Z(f) \cap \mathbb{T}^{3}$ with respect to the pullback of the Lebesgue measure, and which has density zero everywhere else. We illustrate this method through an example. The general proof for flat polynomials is analogous.

Example 1 Consider the polynomial

$$
p\left(z_{1}, z_{2}, z_{3}\right)=2-z_{1} z_{2}-z_{1} z_{3} .
$$

A piece of the zero set of this polynomial is parametrized by $(s,-s,-s)$, and so, the corresponding Cauchy transform is given by

$$
C[\mu](z)=\frac{1}{2 \pi} \int_{0}^{2 \pi} \frac{1}{\left(1-e^{i s} z_{1}\right)\left(1-e^{-i s} z_{2}\right)\left(1-e^{-i s} z_{3}\right)} d s .
$$

By replacing the factors in the integral by their power series expansions and changing orders of integration and summation, we see that

$$
C[\mu]\left(z_{1}, z_{2}, z_{3}\right)=\sum_{l, m \in \mathbb{N}}\left(z_{1} z_{2}\right)^{l}\left(z_{1} z_{3}\right)^{m}=\frac{1}{1-z_{1} z_{2}} \frac{1}{1-z_{1} z_{3}} .
$$

If the above function lies in $D_{-\alpha}$, then, by Lemma $5, p\left(z_{1}, z_{2}, z_{3}\right)$ is not cyclic in $D_{\alpha}$.

We have that $C[\mu]$ lies in $D_{\alpha}(\alpha<0)$ if and only if

$$
\|C[\mu]\|_{\alpha}^{2}=\sum_{l=0}^{\infty} \sum_{m=0}^{\infty}(1+l+m)^{\alpha_{1}}(1+m)^{\alpha_{2}}(1+l)^{\alpha_{3}}<\infty .
$$

The above series is finite if

$$
\sum_{l=0}^{\infty}(1+l)^{\alpha_{3}} \sum_{m=0}^{l}(1+l+m)^{\alpha_{1}}(1+m)^{\alpha_{2}}<\infty .
$$

and the corresponding series where $m \geq l$ is finite. The calculations for both series of the above form are analogous.

Since $l \geq 0$, we have that

$$
\sum_{m=0}^{l}(1+l+m)^{\alpha_{1}}(1+m)^{\alpha_{2}} \leq \sum_{m=0}^{l}(1+m)^{\alpha_{1}+\alpha_{2}} \leq c(1+l)^{\alpha_{1}+\alpha_{2}+1} .
$$

By plugging this into (9), we see that

$$
\sum_{l=0}^{\infty}(1+l)^{\alpha_{3}} \sum_{m=0}^{l}(1+l+m)^{\alpha_{1}}(1+m)^{\alpha_{2}} \leq c \sum_{l=0}^{\infty}(1+l)^{\alpha_{1}+\alpha_{2}+\alpha_{3}+1}
$$


and the last expression is finite if and only if $|\alpha|+1<-1$, which implies that $|\alpha|<-2$.

It follows that $C[\mu] \in D_{\alpha}$ if $|\alpha|<-2$, and so $2-z_{1} z_{2}-z_{1} z_{3}$ is not cyclic for any $\alpha \in(0,1)^{3}$ with $|\alpha|>2$.

Note that the above calculations only depend on the zero set, and really is not using any other property of the polynomial. For example, the corresponding calculations for the polynomial $5-x y-x^{3} y^{3}-x z-2 x^{2} y z$ are exactly the same. Note also that if one tries to carry out the corresponding calculations for the polynomial $2-x-y z$, then the result is that the corresponding series converges if and only if $\alpha>1$, which is in line with Theorem 1.

By applying Frostman's lemma it can be shown that the above calculations are in fact sharp.

Theorem 8 Let $S \subset \mathbb{T}^{3}$ have (Hausdorff) dimension equal to $s \in \mathbb{N}$. Then $S$ has isotropic $\alpha$-capacity equal to zero for all $\alpha \leq 1-s / 3$.

Proof For all $a, b, c \in \mathbb{R}$, we have that

$$
(a b c)^{(2 / 3)} \leq\left(\max \{a, b, c\}^{3}\right)^{(2 / 3)}=\max \{a, b, c\}^{2} \leq a^{2}+b^{2}+c^{2} .
$$

It follows that, for any positive measure $\mu$

$$
\begin{aligned}
& \int_{S} \int_{S} \frac{d \mu(x) d \mu(y)}{\left(\left|e^{i x_{1}}-e^{i y_{1}}\right|\left|e^{i x_{2}}-e^{i y_{2}}\right|\left|e^{i x_{3}}-e^{i y_{3}}\right|\right)^{1-\alpha}} \\
& \quad \geq \int_{S} \int_{S} \frac{d \mu(x) d \mu(y)}{\left(\sqrt{\left|e^{i x_{1}}-e^{i y_{1}}\right|^{2}+\left|e^{i x_{2}}-e^{i y_{2}}\right|^{2}+\left|e^{i x_{3}}-e^{i y_{3}}\right|^{2}}\right)^{3(1-\alpha)}} .
\end{aligned}
$$

By Frostman's lemma (for example Theorem 8.9 in [8]), we know that the right hand side is infinite for every probability measure $\mu$ supported on $S$ if $3(1-\alpha) \geq s$. It follows that $S$ has $\alpha$-capacity zero for all $\alpha \leq 1-s / 3$.

This shows that the estimates in Example 1 and Theorem 5 are sharp in the sense that we could not have pushed non-cyclicity further by selecting different measures supported on these zero sets. If the Brown and Shields conjecture is true, then this would also imply that functions whose zero set is a curve are cyclic in the isotropic Dirichlet type space on the tridisc for all $\alpha \leq 2 / 3$, and functions whose zero set is a hypersurface are cyclic for all $\alpha \leq 1 / 3$.

In two variables, only the cardinality of the zero set was important regarding cyclicity. However, the above example shows us that polynomials in three variables whose zero set is a curve have worse cyclicity properties than those whose zero set is finite, but better than those whose zero set is a hypersurface. It seems like dimension of the zero set, and not only cardinality is important. But furthermore, in two variables, the only deviation of this behaviour was for polynomials which were not truly two variable polynomials. In three variables, we still have this property, but furthermore, we know that even the orientation of the zero set is important, and we have examples of genuine three variable polynomials where this comes into play. 
Acknowledgements The author thanks the referee for a careful reading of the paper, and Alan Sola for several valuable discussions and ideas.

\section{Compliance with ethical standards}

Conflict of interest The author declares that he has no conflict of interest.

Open Access This article is distributed under the terms of the Creative Commons Attribution 4.0 International License (http://creativecommons.org/licenses/by/4.0/), which permits unrestricted use, distribution, and reproduction in any medium, provided you give appropriate credit to the original author(s) and the source, provide a link to the Creative Commons license, and indicate if changes were made.

\section{References}

1. Bénéteau, C., Condori, A.A., Liaw, C., Seco, D., Sola, A.: Cyclicity in Dirichlet-type spaces and extremal polynomials II: functions on the bidisk. Pac. J. Math. 276, 35-58 (2015)

2. Bénéteau, C., Knese, G., Kosiński, Ł., Liaw, C., Seco, D., Sola, A.: Cyclic polynomials in two variables. Trans. Am. Math. Soc. 368, 8737-8754 (2016)

3. Brown, L., Shields, A.L.: Cyclic vectors in the Dirichlet space. Trans. Am. Math. Soc. 285, 269-304 (1984)

4. El-Fallah, O., Kellay, K., Mashreghi, J., Ransford, T.: A Primer on the Dirichlet Space. Cambridge Tracts in Mathematics, vol. 203. Cambridge University Press, Cambridge (2014)

5. Kaptanoğlu, T.H.: Möbius invariant Hilbert spaces in polydiscs. Pac. J. Math. 163(2), 337-360 (1994)

6. Knese, G., Kosiński, Ł., Ransford, T.J., Sola, A.A.: Cyclic polynomials in anisotropic Dirichlet spaces. arXiv:1512.04871.pdf

7. Łojasiewicz, S.: Introduction to Complex Analytic Geometry. Birkhäuser, Basel (1991)

8. Mattila, P.: Geometry of Sets and Measures in Euclidean Spaces. Cambridge Studies in Advanced Mathematics. Cambridge University Press, Cambridge (1995)

9. Stein, E.M.: Harmonic Analysis: Real-Variable Methods, Orthogonality, and Oscillatory Integrals. Princeton University Press, Princeton (1993) 